\title{
Quantification of Atomic Arrangements at Heterostructure Interfaces
}

Donovan N. Leonard ${ }^{1}$, Ondrej Dyck ${ }^{1}$, Jonathan D. Poplawsky ${ }^{1}$, Karren L. More ${ }^{1}$, Lisa F. Edge ${ }^{2}$, Clayton A. Jackson ${ }^{2}$, Emily J. Pritchett ${ }^{2}$, and Peter W. Deelman ${ }^{2}$

1. Oak Ridge National Laboratory, Oak Ridge, TN, USA

2. HRL Laboratories, LLC, Malibu, CA, USA

This study used a combination of atomic-scale characterization techniques to measure interface widths and structures of $\mathrm{Si} / \mathrm{SiGe}$ heterostructure interfaces. The contrast in high angle annular dark field scanning transmission electron microscopy (HAADF-STEM) images was analyzed using column intensity histograms and sigmoid fits to determine atomic-scale interface abruptness and width. HAADF-STEM computational models were also employed to better understand sample thickness effects on sigmoid fitting and how thicker or thinner regions affected the measured interface widths. The $\mathrm{Si} / \mathrm{SiGe}$ interface data extracted from HAADF-STEM images was compared to atom probe tomography (APT) results from the same $\mathrm{Si} / \mathrm{SiGe}$ heterostructure specimens.

A Nion UltraSTEM operated at $100 \mathrm{kV}$ with a beam current $\sim 45 \mathrm{nA}$, was used to interrogate the atomic structure of $\mathrm{Si} / \mathrm{SiGe}$ interfaces. The $\mathrm{Si} / \mathrm{SiGe}$ samples were prepared using both focused ion beam (FIB) and conventional Ar ion beam thinning methods. The Si/SiGe interfaces were imaged in the $<110>$ orientation and Si dimer structures measuring $1.4 \AA$ were clearly resolved. Column intensity histograms from $\sim 1000$ atomic columns were generated from the HAADF-STEM images using Python code modified by the authors. STEM image simulations were performed using Dr. Probe software (freeware) [1]. APT needles were prepared using a standard FIB lift-out technique and field evaporation was performed with a Cameca Instruments Local Electrode Atom Probe (LEAP) 4000X HR.

A typical HAADF-STEM image of the $\mathrm{Si} / \mathrm{SiGe}$ heterostructure interface is shown in Fig. 1. After column intensities were determined, a 2-sigma variation was assigned to the histograms to reveal the atomic column positions and the local interfacial chemistries. Intensities between the 2-sigma distributions, likely resulting from compositions outside of $100 \% \mathrm{Si}$ and $\mathrm{Si}_{0.7} \mathrm{Ge}_{0.3}$, are marked on the HAADF-STEM image with light blue dots and denote atomic columns associated with the interface. Quantification of interface widths was achieved by fitting a sigmoid curve to average HAADF-STEM image intensities (Fig. 2). Interestingly, samples thicknesses of $\sim 20-30 \mathrm{~nm}$ gave the most consistent interface width measurements.

Results from this investigation show that sample thickness skews the sigmoid fitting and affects interfacial width quantification. Previously published methodologies for measuring the atomic-scale interfacial roughness [2-4], and correlation of HAADF-STEM results with APT data (Fig. 3), will also be presented [5].

[1] http://www.er-c.org/barthel/drprobe/

[2] Luna, E., Ishikawa, F. and Batista, P., Applied Physics Letters 92 (2008) p. 141913.

[3] S. M. Goodnick, D. K. Ferry, and C. W. Wilmsen, Phys. Rev. B 32 (1985) p. 8171.

[4] A. Ourmazd, D. W. Taylor, and J. A. Rentschler, Phys. Rev. Lett. 59 (1987) p. 213.

[5] This research was conducted at the Center for Nanophase Materials Sciences, which is a DOE Office of Science User Facility. 

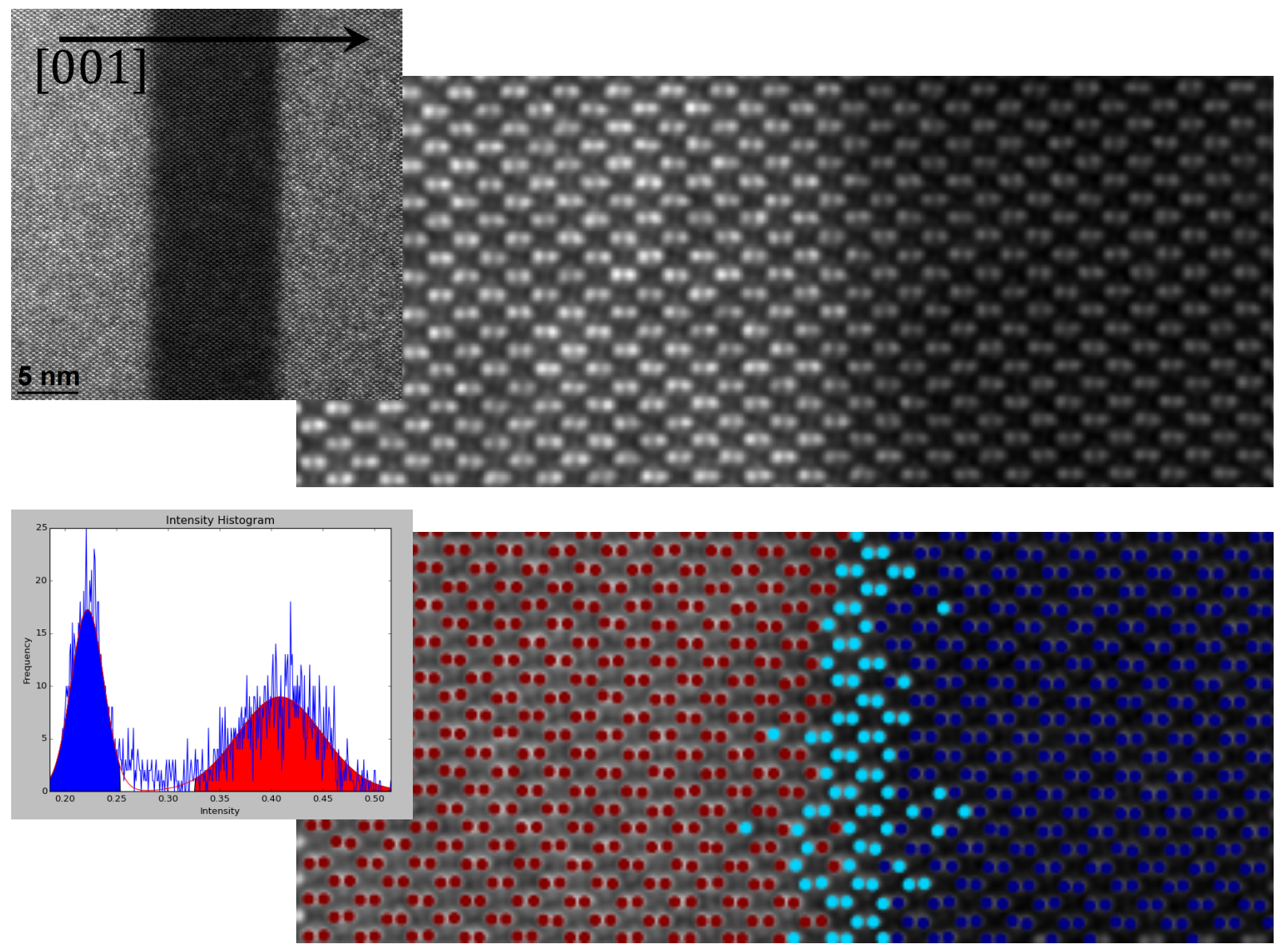

Figure 1. (Top Left) STEM HAADF micrograph showing a $\sim 10 \mathrm{~nm} \mathrm{Si} \mathrm{well} \mathrm{in} \mathrm{between} \mathrm{SiGe} \mathrm{layers.} \mathrm{The} \mathrm{[001]} \mathrm{growth}$ direction is indicated by arrow direction. (Top Right) Atomic resolutionmicrograph showing HAADF-STEM contrast of SiGe (left) and Si dimers (right) at the bottom interface imaged in the $<110>$ orientation. (Bottom Left) Column intensity histogram from HAADF STEM image. (Bottom Right) HAADF overlay showing positions of SiGe columns (red dots), Si columns (blue dots) and mixed interface structure (light blue dots).
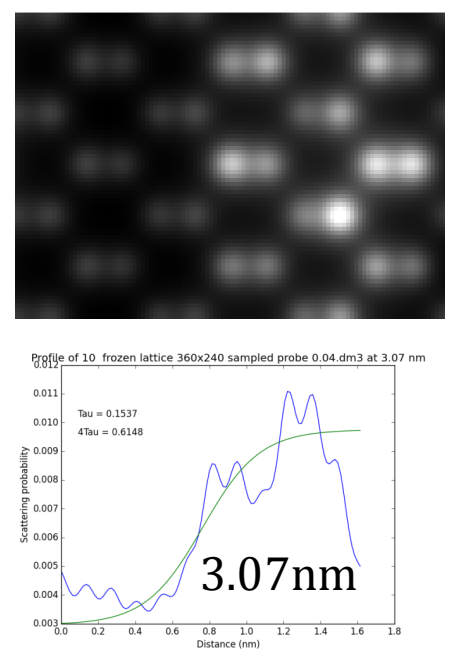


Figure 2. (Left) Simulated STEM HAADF image of $\sim 3 \mathrm{~nm}$ thick sample $\mathrm{Si} / \mathrm{SiGe}$ interface and corresponding sigmoid fit of HAADF intensity profile. (Right) Simulated HAADF image and sigmoid fit of $\sim 21 \mathrm{~nm}$ thick sample.
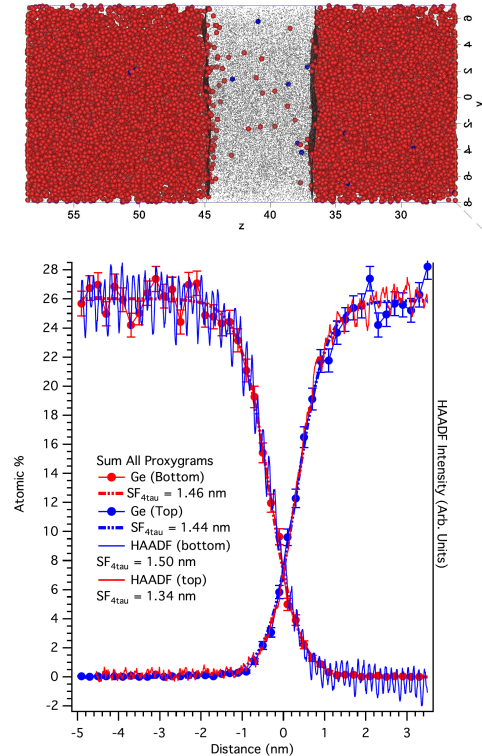

Figure 3. APT data used to compare interface width measurements from STEM HAADF data. 